Ann. Zootech., I973, 22 (3), 253-265.

$$
\begin{aligned}
& \text { I.N.R.A. } \\
& \text { BIBLIOTHEQUE UO } 3593: \\
& \text { DOMAINE DE CROUELLE } \\
& \text { 63039 } \\
& \text { CLERMONT-FD CEDEX }
\end{aligned}
$$

\title{
UTILISATION ÉNERGÉTIQUE ET AZOTÉE DES PRINCIPAUX TOURTEAUX ET DES GRAINES DE LÉGUMINEUSES PAR LE RAT EN CROISSANCE; COMPARAISON AU BLÉ TENDRE ET AU BLÉ DUR
}

\author{
M. VERMOREL \\ aves la collaboration technique de J. Bejot, Françoise Duboisset et J.-C. PAcrot \\ Station d'Étude des Métabolismes, \\ Centre de Recherches de Clermont-Ferrand, I. N.R. A., \\ Saint Genès Champanelle, 63110 Beaumont
}

RÉSUMÉ

En complément des travaux de Y. HenRy chez le Porc, nous avons étudié les valeurs énergétiques et azotées du blé tendre, du blé dur, du pois fourrager, de la féverole et des tourteaux d'arachide, de soja et de tournesol pour le rat en croissance par la méthode des abattages comparés. Ces aliments représentent une proportion aussi élevée que possible de l'énergie et de l'azote des régimes supplémentés en acides aminés indispensables. L'utilisation de ces régimes est comparée à celle d'un régime témoin à base d'amidon et de farine de hareng.

Les digestibilités de l'énergie et de l'azote de ces aliments, leurs énergies nettes rapportées à celles de l'amidon et de l'orge, sont comparées aux valeurs obtenues chez le Porc. L'utilisation métabolique des protéines supplémentées en acides aminés indispensables est variable suivant les aliments, inférieure en particulier dans le cas de la féverole. Pour une même ingestion d'énergie métabolisable, ces différences se traduisent par une modification de la synthèse protéique et de l'engraissement des rats.

\section{I. - INTRODUCTION}

Dans l'alimentation des monogastriques, les céréales constituent l'apport énergétique et azoté de base mais elles doivent être supplémentées par d'autres sources de protéines (tourteaux, graines de légumineuses, farines animales...). Ces aliments représentent également un complément énergétique non négligeable, mais la composition chimique des glucides en particulier, l'utilisation digestive des différentes fractions et leur valeur énergétique nette sont mal connues. En effet, leur concentration dans les régimes est généralement faible (Io à $30 \mathrm{p}$. IOO) et ne permet 
pas de mesures précises; une incorporation plus importante entraîne un déséquilibre des régimes et par suite des résultats discutables.

Pour préciser ces données nous avons entrepris l'étude de la valeur nutritive, énergétique et azotée, des principaux tourteaux utilisés en France, du pois fourrager et de la féverole, susceptibles de se substituer à ces tourteaux. Ces études ont été réalisées chez le Rat en croissance, dont les besoins azotés sont bien connus et voisins de ceux du porcelet, en complément de certains travaux de HENRY et RÉRAT (I969) sur le Porc. Nous avons également étudié une nouvelle variété de blé dur très intéressante du point de vue agronomique, en raison du manque de données sur la valeur nutritive de cette céréale. Nous nous sommes intéressé particulièrement à l'influence de ces aliments, introduits dans des régimes équilibrés, sur l'orientation du métabolisme du rat en croissance (accroissement de la protéinogenèse ou de la lipogenèse) en raison de l'importance de ce phénomène dans la production intensive d'animaux de boucherie.

\section{II. - MATÉRIEL E'T MÉTHODES}

\section{A. - Aliments}

Le blé dur "Agathe " sélectionné par la Station d'Amélioration des Plantes de Montpellier est une nouvelle variété de printemps très productive qui présente une teneur élevée en protéines (tabl. I), et est capable d'assurer la production de protéines la plus élevée à l'hectare pour les céréales. Le blé tendre "Magdaléna ", utilisé comme référence, et les tourteaux d'arachide, de soja et de tournesol sont des aliments d'origine commerciale. Le tourteau de tournesol a été obtenu par pression et présente une teneur assez élevée en huile résiduelle.

Les échantillons de pois fourrager (Pisum arvense) "Moyencourt " et de féverole "Ascott " (Vicia faba) ont été fournis par des sélectionneurs et choisis parmi d'autres variétés pour leurs teneurs en protéines et leurs digestibilités élevées observées au cours d'un premier essai.

\section{B. - Régimes}

Les aliments ont été introduits dans les régimes en proportion aussi élevée que possible (tabl. 2) d'une part pour améliorer la précision et d'autre part pour réaliser une concentration azotée de 14 à $\mathrm{I} 6 \mathrm{p}$. Ioo par rapport à la matière sèche suivant les digestibilités des régimes déterminées préalablement. Le complément de l'apport énergétique a été assuré par de l'amidon de blé. La composition en acides aminés des protéines a été déterminée par chromatographie échangeuse d'ions (PION et FAUCONNEAU, 1966) ou estimée à partir de celles d'aliments comparables déjà analysés (PION, I97I). La supplémentation azotée des aliments a été réalisée à l'aide d'acides aminés industriels pour satisfaire les besoins en acides aminés indispensables du Rat en croissance précisés par PAwLAK et PION (1968, I97I). L'apport d'acides gras essentiels a été assuré par 2 p. Ioo d'huile de maïs.

Pour éviter les accidents observés avec certaines graines de légumineuses consommées en quantités importantes, le pois fourrager et la féverole ont été cuits à l'air libre pendant une heure environ après broyage et addition d'eau. Les régimes, présentés sous forme de pâtée liquide, ont été distribués ad libitum aux animaux et les refus ont été pesés chaque jour.

\section{C. - Animaux}

Des rats Wistar mâles ont été répartis en 7 lots comparables de 18 animaux, au poids moyen de $75 \mathrm{~g}$. Un des lots a été abattu en début d'expérience et la composition corporelle moyenne a été déterminée. Les rats de 6 autres lots ont reçu les régimes expérimentaux pendant une durée de 30 jours.

Lors du dépouillement des résultats nous avons été gênés pour la détermination de la valeur 


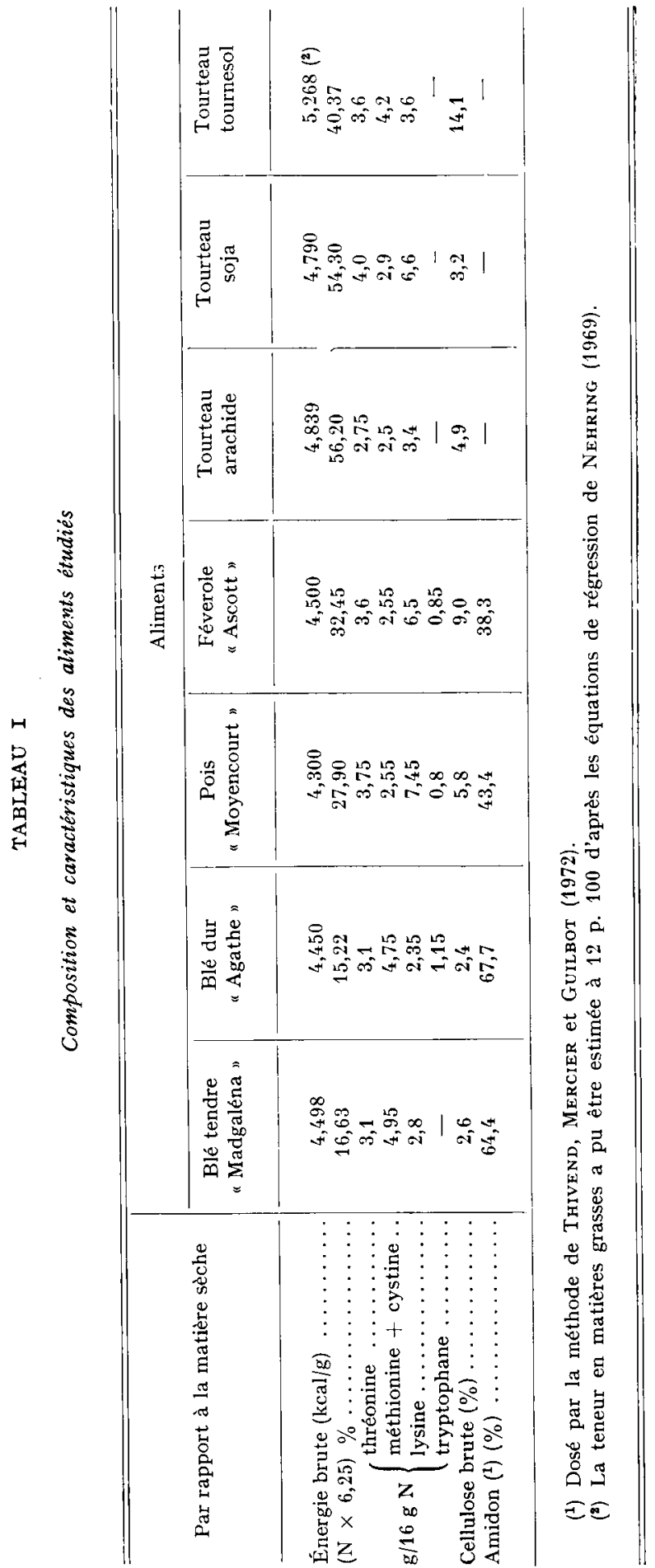


M. VERMOREI

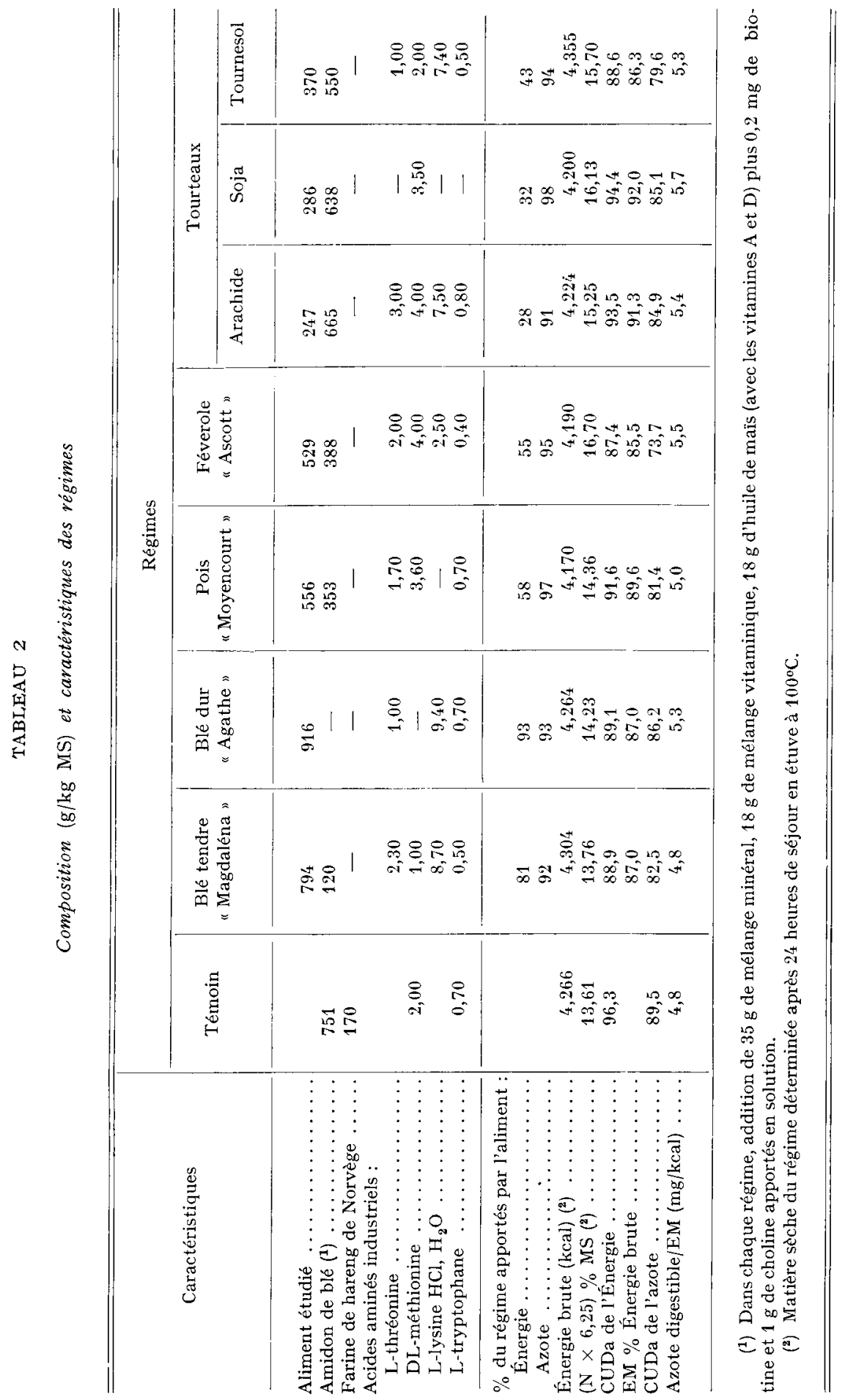


énergétique des aliments par l'absence d'un lot de rats ayant reçu de l'amidon. Aussi, l'année suivante à la même époque nous avons constitué 2 autres lots de rats comparables aux précédents : le premier a été abattu à $75 \mathrm{~g}$, le second a reçu le régime témoin pendant une durée de 30 jours et selon une courbe de consommation comparable à celles des autres lots.

$$
\text { D. - Mesures }
$$

\section{Gains de poids vif.}

Les animaux ont été pesés 2 fois par semaine, à heure fixe le matin avant la distribution de la ration, au début de l'expérience et le jour de l'abattage.

Digestibilités. - Au cours de deux autres essais, les digestibilités apparentes de l'énergie et de l'azote des régimes ont été déterminées sur io rats en croissance, pendant une période de collecte de Io jours, après adaptation. En raison de la variabilité réduite du CUD entre rats $(0,5$ p. Ioo pour l'énergie et I,2 p. Ioo pour l'azote d'après nos résultats précédents) les fèces des animaux qui présentaient des consommations régulières et voisines ( 7 à 8 par régime) ont été mélangées, lyophilisées puis broyées. Nous avons également mélangé les échantillons d'urine. Les autres animaux n'ont pas été pris en considération.

Les CUD de l'énergie et de l'azote des aliments ont été calculés à partir de ceux des régimes en estimant à 98 le CUD de l'amidon et de l'huile de maïs et à roo celui des acides aminés industriels, (valeurs déterminées avec des régimes témoins à base d'amidon et de farine de poisson).

Préparation des échantillons. - En fin d'expérience les rats ont été sacrifiés, le contenu digestif éliminé et les carcasses pesées avec précision. Elles ont ensuite été congelées puis broyées individuellement à l'aide d'un appareil Robotcoupe et la teneur en matière sèche a été déterminée en triple, pendant $24 \mathrm{~h}$ à $10^{\circ} \mathrm{C}$. Deux parties aliquotes (de $3^{\circ} \mathrm{g}$ environ) du broyat de chaque carcasse ont été prélevées et traitées séparément. L'ensemble des prélèvements correspondants des animaux d'un même lot a été réhomogénéisé en vue de constituer un échantillon moyen. Une prise de 120 g environ de cet homogénat a été mélangée dans un mixer avec un poids égal d'eau . Des prélèvements de 4 à $6 \mathrm{~g}$ de ce mélange ont été effectués dans des sachets en polythène (I $20 \mathrm{mg}$ ) et lyophilisés. brute.

Les fèces ont été lyophilisées ainsi que les urines en vue de la détermination de leur énergie

Analyses. _- L'énergie brute a été mesurée à l'aide d'un calorimètre adiabatique et la teneur en azote déterminée par la méthode macrokjeldahl. Les dosages ont été effectués en triple, c'està-dire en sextuple pour un lot de carcasses dont 2 échantillons ont été préparés séparément.

Interprétation des résultats. — L'interprétation statistique des résultats de l'expérimentation en lots a été faite par analyse de variance et comparaison des moyennes à l'aide du test de Tukey en estimant la variabilité intra-lot : pour les teneurs en énergie et en azote des carcasses nous avons retenu le coefficient de variation de 6 p. roo que nous avons déterminé précédemment au cours d'expériences comparables. L'énergie nette des aliments pour l'ensemble " entretien + croissance) est comparée à celle de l'amidon en calculant quelle quantité du régime témoin aurait été nécessaire pour assurer la même fixation d'énergie que celles des autres lots (AURoussEAU, DE GrooT et VERMOREL, I970).

\section{III. - RÉSULTA'TS}

\section{A. - Digestibilités}

Les caractéristiques des aliments étudiés sont indiquées dans les tableaux I et 4 , la composition et les digestibilités des régimes dans le tableau 2. Les aliments représentent une partie importante des régimes, $9 \mathrm{I}$ à $98 \mathrm{p}$. Ioo de 1'azote, 55 et 58 p. roo de l'énergie pour les graines de légumineuses, 28 à $43 \mathrm{p}$. roo pour les tourteaux.

- Les CUD de l'énergie et de l'azote du blé dur "Agathe " sont supérieurs à ceux des blés tendres que nous avons étudiés par ailleurs, ce qui est à mettre en relation, dans le cas de l'énergie, avec une teneur en amidon légèrement supérieure à celle du blé « Magdaléna ». Les CUD de l'énergie et de l'azote du pois " Moyencourt " 
sont égaux à ceux du blé tendre et supérieurs de 8 points à ceux de la féverole "Ascott ". De même, si les digestibilités des tourteaux d'arachide et de soja sont élevées et comparables, celle du tourteau de tournesol est inférieure de 5 à 7 points. Compte tenu de l'énergie brute élevée des tourteaux, l'énergie métabolisable (EM) des 2 blés et des 3 tourteaux est voisine $(3,83$ à $3,87 \mathrm{kcal} / \mathrm{g} \mathrm{MS})$, celles du pois et de la féverole étant inférieures de 5 et Io $\mathrm{p}$. Ioo respectivement.

\section{B. - Utilisation métabolique}

Les principaux résultats de l'expérimentation en lots sont groupés dans le tableau 3. Les quantités de E,M moyennes ingérées par les 8 lots sont voisines (I goo $\pm 38 \mathrm{kcal}$ ), ce qui facilite l'interprétation des résultats. Les gains de poids vif moyens sont légèrement plus élevés pour le lot " tournesol " et légèrement plus faibles pour les lots " blé dur " et " féverole ", mais aucune différence n'est significative.

\section{Utilisation des protéines.}

Les quantités de protéines fixées par les rats des lots "Blé dur " et "Féverole » sont significativement inférieures à celles des autres lots : de $5 \mathrm{p}$. Ioo par rapport aux lots "Témoin ", "Blé tendre " et "Soja " et de 7 p. Ioo par rapport au lot "Tournesol ". Le CUP de l'azote du régime "Féverole " est significativement inférieur à celui des autres régimes, en raison du CUD particulièrement faible et du coefficient de rétention azotée ( $\mathrm{CRa}$ ) significativement inférieur à celui des régimes "Blé tendre", " Témoin ", "Pois ", et " Tournesol ". Les CRa des régimes "Soja ", "Arachide " et "Blé dur " sont également significativement inférieurs à ceux des 4 régimes précédents.

\section{Utilisation de l'énergie.}

Les quantités d'énergie fixée par les rats des lots " Témoin " et "Blé dur " sont significativement supérieures à celles des autres lots. Cependant les quantités de EM ingérée ne sont pas identiques et pour tenir compte de ces différences nous avons estimé à $7 \mathrm{I}$ p. Ioo l'utilisation de EM pour la croissance, d'après nos résultats précédents obtenus dans la même zone de croissance (VERMOREL et KELLER, I967). Pour une même ingestion de EM (Igoo kcal) l'énergie fixée a été en valeur relative pour les différents régimes : "Témoin " Ioo, "Blé dur " 95, "Tournesol " 90, "Soja " 89, "Blé tendre " 87, "Pois " 86,6, "Féverole " 85, "Arachide " 82.

Si l'on admet que les faibles différences de EM ingérée ( \pm 2 p. Ioo) n'ont pas une incidence très importante $(2$ à $3 \mathrm{p}$. Ioo) sur la fixation de protéines, on peut calculer par différence que pour une même ingestion de EM, les lots "Témoin " et "Blé dur " ont fixé plus de lipides que les autres lots, respectivement 22 et I 8 p. Ioo de plus que les lots "Soja » et "Tournesol ", 26 et 22 p. Ioo de plus que les lots " Pois », "Féverole " et "Blé Magdaléna ». Cet engraissement supérieur des rats des lots "Témoin " et "Blé dur " se traduit à la fois par une teneur en matière sèche des carcasses significativement plus élevée et par un pourcentage d'énergie fixée sous forme de protéines significativement inférieur à ceux des autres lots, les carcasses des lots "Féverole ", " Pois " et "Tournesol " étant à cet égard les plus maigres.

Le calcul de l'énergie nette des échantillons d'aliments étudiés pour l'entretien et la croissance, par rapport à l'amidon, met en évidence la valeur élevée du blé dur 
U'TILISATION DES ALIMENTS PAR LE RAT

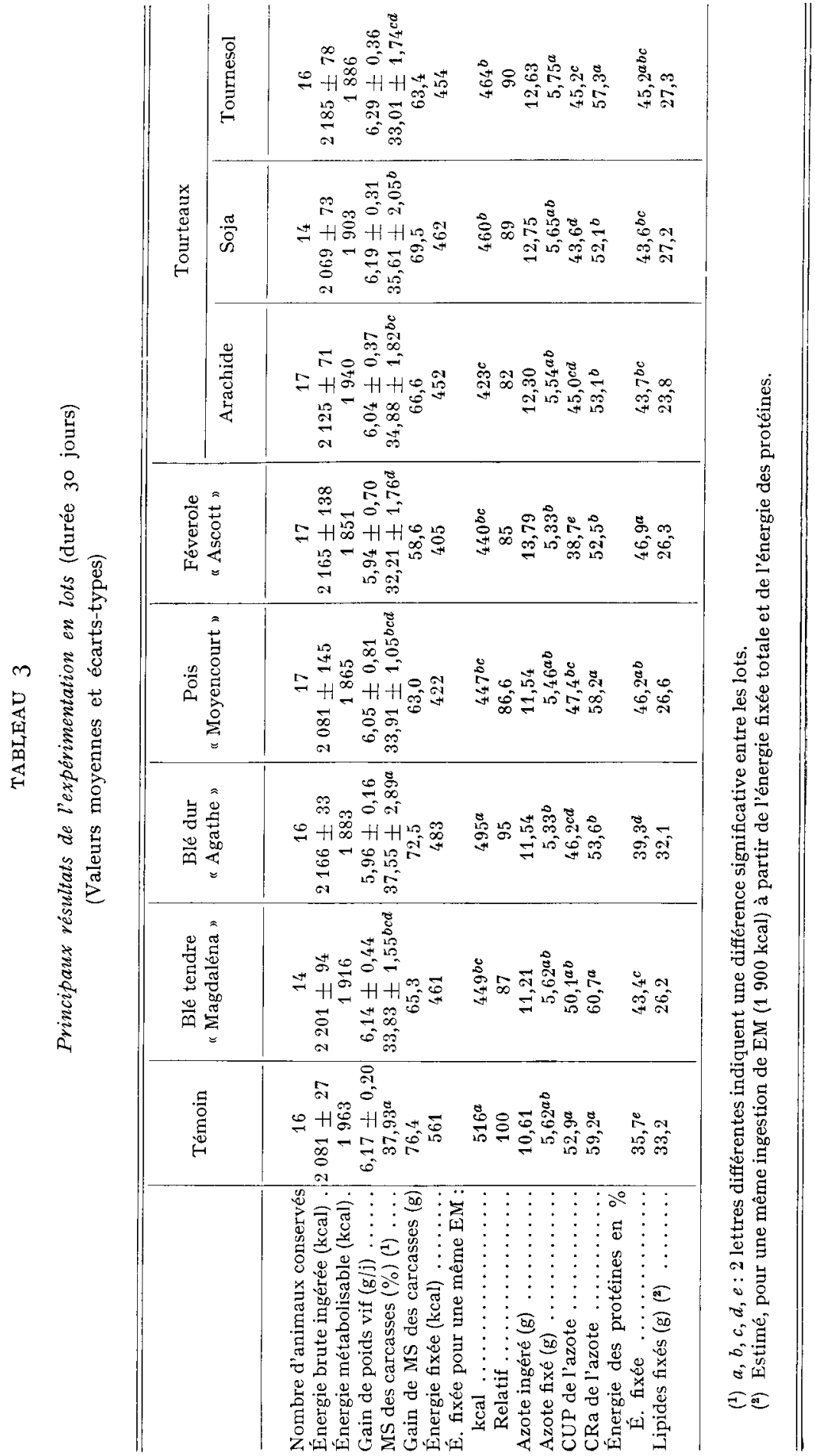


"Agathe ", supérieure de 6 à 7 p. Ioo à celles du blé tendre et des tourteaux de soja et de tournesol. En revanche, l'énergie nette de la féverole et celle du tourteau d'arachide sont beaucoup plus faibles. Le rendement de EM en énergie nette est, en valeur relative, pour les aliments étudiés : amidon et blé dur : Ioo, blé tendre : 95,6, tourteau de soja : 93,3, de tournesol : 93,7, pois : 93,4, féverole : 90,o, tourteau d'arachide 78,5 .

\section{IV. - DISCUSSION ET CONCLUSION}

\section{A. - Conditions expérimentales}

Les proportions élevées d'énergie et d'azote apportées par les aliments étudiés dans des régimes équilibrés en acides aminés indispensables nous permettent d'espérer une meilleure précision dans l'estimation de leur énergie nette ainsi qu'une bonne appréciation de l'utilisation métabolique des acides aminés et de leur influence sur l'orientation du métabolisme. En effet, on peut calculer dans le cas du tourteau d'arachide, qui n'apporte que $28 \mathrm{p}$. Ioo de l'énergie du régime, qu'une erreur de 2 p. Ioo dans le bilan énergétique entraîne une erreur de $4 \mathrm{p}$. Ioo sur l'énergie nette de ce tourteau.

En revanche, le fait que le régime témoin, à base d'amidon et de farine de poisson, n'ait pas été étudié en même temps que les autres est un inconvénient pour l'interprétation des résultats. En effet, on sait qu'avec un même régime les résultats peuvent varier d'un essai à l'autre, en particulier la rétention azotée ; dans notre cas, les rats provenaient du même élevage et ont été pris au même poiđs et à la même période de l'année. Le lot "Blé tendre Magdaléna » est également un " témoin 》 valable pour les autres lots.

Il est intéressant de noter que si les gains de poids vifs moyens ne sont pas significativement différents, il existe, en revanche, des différences hautement significatives au niveau de la rétention azotée, de l'énergie fixée et de l'état d'engraissement, ce qui confirme que le gain de poids vif est un critère très insuffisant pour des études de ce type.

\section{B. - Rendement de l'énergie métabolisable}

Le rendement de l'EM des aliments en énergie nette baisse en fonction de 2 facteurs qui ne sont peut-être pas indépendants : d'une part la diminution de la teneur en amidon et d'autre part l'augmentation de la quantité de protéines formées et du pourcentage d'énergie fixée sous forme de protéines. Ces observations confirment le fait que l'obtention de carcasses maigres a lieu au prix d'un rendement moindre de l'E,M. Ce phénomène peut avoir entre autres causes, d'une part le coût énergétique élevé de la protéinogenèse apparente, supérieur à celui de la lipogenèse apparente (SchiEmanN, I970), d'autre part le rendement énergétique inférieur de certains nutriments (comme les acides gras volatils) formés au cours de la digestion des glucides autres que l'amidon et qui ont une action lipogénétique moindre que l'amidon. Cette dernière hypothèse repose sur les observations faites dans le cas des légumineuses et des tourteaux, des farines de luzerne (VERMOREL et PION, I973) et de l'acide acétique (VERmorei, et Aurousseau, I970). 


\section{C. - Valeurs énergétique et azotée des aliments étudiés. Comparaison avec les données relatives au Porc}

Au cours d'une expérience comparable (VERMOREL, I973) nous avons déterminé l'énergie nette de diverses céréales, en particulier celles d'un blé tendre et d'une orge. Ces données nous permettent de calculer la valeur énergétique nette de ces 7 aliments par rapport à une orge moyenne en vue de comparer nos résultats à ceux obtenus par d'autres auteurs chez le Porc (tabl. 4).

Le blé dur "Agathe " est riche en protéines qui présentent les mêmes carences en acides aminés indispensables que les blés tendres ; la teneur en lysine est inférieure à celle du blé "Magdaléna " ou d'autres blés tendres de teneurs en azote voisines (Pion et Fauconneau, I969). De plus, malgré la supplémentation en acides aminés et une concentration azotée plus élevée $(5,3$ contre $4,8 \mathrm{mg} \mathrm{Nd} / \mathrm{kcal} \mathrm{E} / \mathrm{M}), 1$ 'utilisation métabolique des protéines du blé dur est inférieure à celle du blé tendre, provoquant une rétention azotée inférieure et par suite un engraissement plus important des animatux.

En revanche, la digestibilité de l'énergie est élevée et l'utilisation de l'EM pour l'entretien et la croissance est supérieure à celle du blé " Magdaléna ", ce qui se traduit par une énergie nette supérieure de $5 \mathrm{p}$. Ioo, en dépit d'une énergie brute légèrement plus faible. Ce phénomène peut résulter à la fois de la teneur plus élevée en amidon et de la protéinogenèse légèrement réduite. On peut donc envisager l'utilisation du blé dur "Agathe " dans l'alimentation des monogastriques moyennant une supplémentation en acides aminés indispensables. Chez le porc cependant il est souhaitable d'étudier son influence sur l'engraissement.

Le pois fourrager "Moyencourt " est riche en protéines qui présentent, comme dans les autres variétés, une teneur élevée en lysine mais faible en acides aminés soufrés. L'utilisation digestive de l'énergie et de l'azote est élevée, chez le Rat comme chez le Porc et comparable à celle du blé. Compte tenu de la teneur assez basse en amidon (43 p. Ioo) et d'un pourcentage non négligeable de cellulose brute ( $6 \mathrm{p}$. IOo), cette valeur élevée du CUDa de l'énergie laisse penser que la cellulose brute du pois est plus digestible que celle de l'orge et que la digestibilité des autres glucides (estimés à 18 p. Ioo de la matière sèche) est importante.

L'énergie nette du pois "Moyencourt " est égale à celle d'une orge moyenne et inférieure de 7 p. Ioo à celle du blé «Magdaléna " essentiellement en raison de la différence d'énergie brute $(4,5 \mathrm{p}$. IOo), 1'utilisation de EM étant inférieure de $2 \mathrm{p}$. Ioo seulement. Ces valeurs relatives sont en accord avec les données des Tables hollandaises (I970) et les résultats de BELI, et WILSON (I970) pour le Porc. L'utilisation métabolique des protéines rééquilibrées du pois est bonne puisqu'elle permet (avec $56 \mathrm{p}$. Ioo de pois dans le régime) une croissance et une formation de protéines non significativement différentes de celles des lots "Blé Magdaléna » et "Témoin », et l'obtention d'animaux plus maigres. Ces performances sont en accord avec les résultats de Bourdon et HENRY (I973) sur le porc en finition et soulignent l'intérêt de cette graine de légumineuse comme source de protéines pour la complémentation des céréales en substitution des tourteaux.

La féverole "Ascott" a une teneur élevée en matières azotées mais ses protéines sont un peu moins riches en lysine et aussi pauvres en acides aminés soufrés que celles 


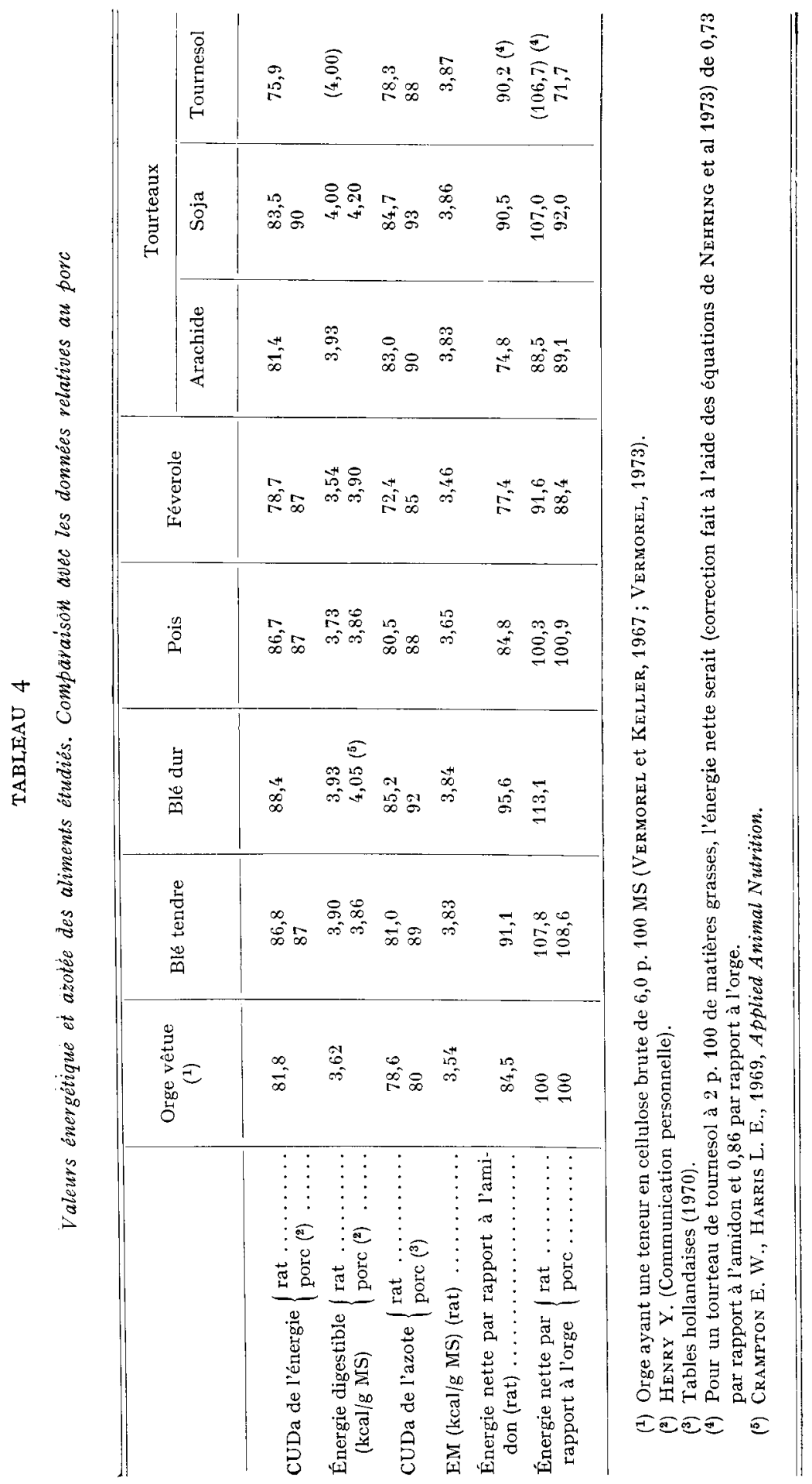


du pois. Le CUDa de l'énergie est inférieur de 8 points à celui du pois ; cette différence peut s'expliquer par les teneurs inférieures en amidon $(-5$ points $)$ et supérieure en cellulose brute $(+3$ points) mais également par le CUDa plus faible des matières azotées ( -8 points). La concentration élevée en cellulose brute peu digestible d'une part et la présence de quantités importantes de tanins (TANGUY et MARTIN, I972) sont susceptibles de réduire le CUDa de l'azote par le biais de l'augmentation de l'azote métabolique fécal ; les résultats obtenus avec le sorgho (VERMOREL, TANGUY et MARTIN, I973) laissent penser que les tanins peuvent jouer un rôle important dans cette diminution du CUDa de l'azote.

Les résultats concernant la digestibilité de l'énergie sont en accord avec ceux de Aherne et de MaC AleEse (I964) sur le Porc et avec les données des tables hollandaises pour le Porc (I970) mais ils sont très inférieurs au CUD déterminé par HENRY et BouRdon (I973) sur le Porc, tant en valeur absolue que par rapport au tourteau de soja ou au pois (BELL et WILSON, I970). On peut cependant remarquer que la digestibilité de la cellulose et des hémicelluloses est plus élevée chez le Porc que chez le Rat (KEys, VAN SoEST et YounG, 1970) et que, par ailleurs, notre régime comportait une proportion plus élevée de féverole que les régimes des porcs.

La féverole présente également une moins bonne utilisation métabolique de l'énergie (rendement de FiM) et de l'azote (CRa) que le pois ; elle n'est probablement pas indépendante de la teneur élevée en cellulose brute et de la présence de tanins qui peuvent être à l'origine d'une importante excrétion fécale d'acides aminés indispensables (Vermores, Tanguy et Martin, r973). Pour ces diverses raisons, d'une part l'énergie nette de la féverole "Ascott " est inférieure de I5 p. Ioo à celle du blé tendre et de $8 \mathrm{p}$. Ioo à celle de l'orge moyenne (ce qui correspond approximativement aux données des Tables hollandaises pour les porcs (1970)), et de 9 p. Ioo à celle du pois "Moyencourt " malgré une énergie brute supérieure de 5 p. Ioo ; d'autre part la rétention azotée est significativement inférieure à celle du pois et du blé tendre. Ces résultats soulignent l'intérêt des travaux sur les méthodes de décorticage de la féverole et sur l'utilisation digestive et métabolique de la graine décortiquée.

Dans le cas des tourteaux, l'énergie nette doit être discutée en fonction de l'énergie brute qui admet d'assez larges variations avec la teneur en matière grasse. Ainsi, le tourteau de tournesol-pression que nous avons étudié avait une énergie brute particulièrement élevée; nous pouvons estimer à $\mathrm{I} 2 \mathrm{p}$. Ioo sa teneur en matières grasses d'après les équations de régression de NeHRING (I969).

Le tourteau de tournesol présente des CUDa de l'énergie et de l'azote faibles, en raison probablement de sa forte teneur en cellulose brute et le problème d'un décorticage plus complet de la graine se pose. Son énergie nette est cependant élevée, égale à celles du blé tendre et du tourteau de soja en raison de son énergie brute importante. A l'aide des équations de régression établies par NEHRING et al. (I963) chez le Rat, on peut calculer que la valeur énergétique nette de ce tourteau, ramené à $2 \mathrm{p}$. Ioo de matières grasses, serait de 0,73 par rapport à l'amidon, soit de 0,86 par rapport à une orge moyenne. Cette valeur est encore très supérieure aux données des Tables hollandaises (I970) pour les porcs (tabl. 4) dans le cas d'un tourteau à I5,6 p. Ioo de cellulose brute. 
Le tourteau de soja a une énergie nette élevée, son énergie brute et sa composition étant tout à fait normales. Selon NEHRING et al. (I963), l'énergie nette d'un tourteau de soja comparable au nôtre est inférieure de 24 p. Ioo à celle de l'orge chez le Rat. Notons que dans cette expérience les protéines ont été utilisées pour la lipogenèse puisque la ration de base couvrait largement les besoins azotés des rats. D'après les Tables hollandaises (I970) pour les porcs, l'énergie nette d'un tourteau de soja comparable au nôtre est inférieure de 8 p. Ioo à celle de l'orge, soit de I4 p. Ioo par rapport au nôtre.

Le tourteau d'arachide, malgré une énergie brute et un CUDa de l'énergie voisins de ceux du tourteau de soja, a une énergie nette inférieure de I 8 p. roo, soit une valeur inférieure de $\mathrm{I} 2 \mathrm{p}$. Ioo à celle de l'orge. Ce résultat correspond à celui de NEHring et al. (I963) chez le Rat et aux données des Tables hollandaises pour le Porc (I970). Nous pensons cependant qu'une partie de l'écart observé par rapport au tourteau de soja peut s'expliquer par des erreurs expérimentales qui ont une répercussion importante puisque le tourteau d'arachide ne fournissait que $28 \mathrm{p}$. Ioo de l'énergie du régime.

Mis à part le faible CUDa de 1'azote du tourteau de tournesol les protéines supplémentées des 3 tourteaux sont bien utilisées, permettant une formation de protéines égale ou supérieure à celle observée avec le régime témoin et l'obtention d'animaux plus maigres. Il faut cependant noter que la concentration azotée des régimes est plus élevée que celle du témoin (5,3 à 5,7 contre 4,8 mg d'azote digestible/kcal EM).

Reçu pour publication en mars 1973.

\title{
SUMMARY
}

\author{
ENERGY AND PROTEIN VALUE OF CURRFNT OIL-MEALS \\ AND LEGUME SEEDS AS COMPARED TO SOFT AND HARD WHEAT
}

IN THE GROWING RAT

The energy and protein value of soft wheat, hard wheat, field-peas, horse beans, peanut, soybean and sunflower oil meals (tabl. I) were determined in the growing rat by the comparative slaughter technique. These feeds were introduced into the balanced diets, supplemented with essential amino acids, in proportions as high as possible : $9 \mathrm{I}$ to $98 \mathrm{p}$. Ioo of the crude protein, $8 \mathrm{I}$ to $93 \mathrm{p}$. Ioo of the energy in the case of wheat, 55 and $58 \mathrm{p}$. Ioo for legume seeds, 28 to $43 \mathrm{p}$. Ioo for oil meals (tabl. 2). The diets were compared to a control diet containing starch and herring meal.

The net efficiency of the metabolizable energy (ME) of the feeds decreased together, with a reduction of the starch content in the food and an increase of the amount of nitrogen retained in the body. The net energy of the feeds as related to that of starch (roo) was the following: soft wheat, 9I.I ; hard wheat, 95.6 ; field-peas, 84.8 ; horse beans, 77.4 ; peanut oil-meal, 74.8 ; soybean oil-meal, 90.5; sunflower oil-meal (rich in lipids), 90.2. These values have been compared with those found in the pig (tabl. 4).

After supplementation with essential amino acids, the metabolic utilization of the proteins of soft wheat, field-peas and oil-meals was good and resulted in a high nitrogen retention and a lower fatness of the animals (tabl. 3). On the other hand, the digestive and metabolic utilization of the supplemented proteins of horse-beans was lower than that of the other feeds, probably because of the high amounts of cell-wall carbohydrates and tannins in the hull of the seed. 


\section{RÉFÍRENCES BIBL,IOGRAPHIQUES}

Aherne F. X., McAleese D. M., I964. Evaluation of tick beans (Vicia faba L.) as a protein supplement in pig feeding. Sci. Proc. Roy. Dublin Soc. I B, II 3 -I 21.

Aurousseau B., de Groot L., Vermorel M., i97o. Étude comparée de l'utilisation énergétique de régimes riches en acide caprylique ou en acides gras insaturés. Ann. Biol. anim. Bioch. Biophys., 10, 703-706.

Bell J.-M., Wilson A. G., I97o. An evaluation of field peas as protein and energy source for swine rations. Canad. J. Anim. Sci., 50, 15-23.

Bourdon D., HenRy Y., I973. Valeur énergétique du pois fourrager et son utilisation par le porc en finition. Journées de la Recherche Porcine en France. I. N. R. A., Paris, I. T. P., éd., Ir 5-12I.

Crampton E. W., Harris L. E., i969. Applied Animal Nutrition, and. rd, p. 753, W. H. Freeman and Co, San Francisco.

Henry Y., Bourdon D., 1973. Utilisation digestive de l'énergie et des matières azotées de la féverole sous forme entière ou décortiquée, en comparaison avec le tourteau de soja. Journées de la Recherche Porcine en France. I. N. R. A., I. T. P., éd. Paris, 105-II4.

Henry Y., Rérat A., I 969 . Utilisation des légumineuses par le Porc en croissance. Journées de la Recherche Porcine en France, I6I-I68, I. N. R. A., Paris, I. T. P. éd.

Keys J. E., Van Soest P. J., Young E. P., xo7o. Comparative study of the digestibility of forage cellulose and hemicellulose in ruminants and non ruminants. J. Anim. Sci., 29, II-I5.

NEHRING K., I 969 . Investigations on the scientific basis for the use of net energy for fattening as a measure of feed value. In : Energy Metabolism of farm Animals. Oriel Press, 5-2o.

Nehring K., Schiemann R., Hoffmann L., Jentsch W., Ig63. Die energetische Verwertung der Futterstoffe. I. Die energetische Verwertung der Kraftfutterstoffe durch Ratten. Arch. Tierernähr, 13, II9-I32.

Pawlak M., Pion R., ig68. Influence de la supplémentation des protéines de blé par des doses croissantes de lysine sur la teneur en acides aminés libres du sang et du muscle du Rat en croissance. $A n n$. Biol. anim. Bioch. Biophys., 8, 5 17-530.

PAWLAK M., PION R., I97I. Influence de la teneur en isoleucine du régime sur la croissance et la teneur en isoleucine libre du sang et du muscle du Rat. Ann. Biol. anim. Bioch. Biophys., 11, 505-5Io.

Pion R., I97I. Composition en acides aminés des aliments. Ind. Alim. Anim., 6, 29-36.

Pion R., Fauconneau G., ig66. Les acides aminés des protéines alimentaires. Méthodes de dosage et résultats obtenus. Cahier de l'A. E. C., nº 6, Commentry 158-175.

Pion R., Fauconneau G., 1969. Composition et valeur nutritive du grain de blé. Bull. Tech. Inf., 244, I-9.

Schiemans R., 1970. Der Energiebedarf für den Eiweissansatz. Wiss. Ztschr. Humboldt Univ. Berlin Math. Naturteiss R., 19, 35-43.

Tanguy J, Martin C., I972. (Communication personnelle).

Thivend P., Mercier Ch., Guilbot A., r972. Determination of starch with glucoamylase. In Whistler R. L., Miller J. N. B. Methods in Carbohydrate Chemistry, 100-105. Academic Press London.

VeEvoedertabel, 1970. Centraal veevoederbureau in Nederland Bornsesteeg 45. Wageningen.

VERMOREL M., 1973. Utilisation énergétique et azotée de nouvelles variétés de céréales par le Rat en croissance (en préparation).

Vermorel M., Aurousseau B., ig7o. Utilisation énergétique des acides acétique et caprylique par le rat en croissance. In : Schurch A., WEN K C., Energy Metabolism of farm animals. Proc. 5th symposium, Vitznau. Schwitzerland, I85-188.

VERMOREL M., KELLER J., I967. Utilisation énergétique par le rat en croissance des principales céréales composant des régimes isoazotés et équilibrés en acides aminés. Ann. Zootech., 16, 223-234.

Vermorel M., Pion R., I973. Valeur énergétique et azotée des luzernes déshydratées. Étude de leur utilisation pour le Rat en croissance. (en préparation).

Vermorel M., Tanguy J., Martin C., r973. Influence de la teneur en tanins sur l'utilisation digestive de l'énergie et de l'azote du sorgho. (en préparation.) 